\title{
Spatial expression of claudin family members in various organs of mice
}

\author{
INHO HWANG $^{1}$, HYUN YANG $^{1}$, HONG-SEOK KANG ${ }^{1}$, CHANG-HWAN AHN $^{1}$, \\ GEUN-SHIK LEE ${ }^{2}$, EUI-JU HONG ${ }^{1}$, BEUM-SOO AN $^{3}$ and EUI-BAE JEUNG ${ }^{1}$ \\ ${ }^{1}$ Laboratory of Veterinary Biochemistry and Molecular Biology, College of Veterinary Medicine, \\ Chungbuk National University, Cheongju, Chungbuk 361-763; ${ }^{2}$ Laboratory of Veterinary Physiology, \\ College of Veterinary Medicine, Kangwon National University, Chuncheon, Gangwon 200-701; \\ ${ }^{3}$ Department of Biomaterials Science, College of National Resources and Life Science, \\ Pusan National University, Miryang, Pusan 627-706, Republic of Korea
}

Received June 22, 2013; Accepted February 17, 2014

DOI: $10.3892 / \mathrm{mmr} .2014 .2031$

\begin{abstract}
Claudins (CLDNs) are tetraspan transmembrane proteins, which are components of tight junctions. The CLDN family is composed of 27 members that are responsible for paracellular transport and certain CLDNs form charge-selective ion channels. CLDNs have two extracellular loops, and the charge of the first extracellular loop determines the ion selectivity of each CLDN. Although the expression and function of each CLDN have been previously investigated, the distribution of CLDNs in various target organs remains to be determined. In the present study, the tissue-specific mRNA distribution of CLDNs (1-5, 7-8, 10a and b, 11-12, 14-17 and 19) in the duodenum, ileum, colon, kidney, liver and lung were defined. Among the tested CLDNs, CLDN1, 2, 12 and 16 were selected for further investiagtion. It was observed that CLDN1, CLDN2 and CLDN12 transcripts and proteins were particularly abundant in the investigated organs. Notably, immune-reactive CLDN16 was detected in a tissue-specific manner and shown in the renal tubules and portal vein. The tested CLDNs were localized to intercellular apical junctions in the epithelium of the intestine, renal tubule and bronchus. Based on this novel information, the presence of several types of CLDNs is of interest as CLDNs may promote or dampen the paracellular diffusion of specific ions.
\end{abstract}

\section{Introduction}

Claudins (CLDNs) are tetraspan membrane proteins consisting of two extracellular loops protruding into the

Correspondence to: Professor Eui-Bae Jeung, Laboratory of Veterinary Biochemistry and Molecular Biology, College of Veterinary Medicine, Chungbuk National University, 52 Naesudongro, Cheongju, Chungbuk 361-763, Republic of Korea E-mail: ebjeung@chungbuk.ac.kr

Key words: claudin, mice intercellular space. The CLDN family consists of at least 27 members. Each type of CLDN has a different expression pattern and function according to tissue type. In structure of CLDNs, the first extracellular loop determines the ion selectivity of CLDNs; sealing, anion- or cation-selective channel (1-6). Certain CLDNs (CLDN1, 3, 5, 11 and 19) contain a non-charged first loop and thus do not exhibit ion selectivity, therefore, they function to seal intercellular junctions. These CLDNs are expressed in tight epithelial cells, including in the skin, rather than in leaky epithelial cells.

CLDNs in tight junctions accumulate within epithelial or endothelial cells that are critical for paracellular transport indicating a novel function of CLDNs as channel proteins. These functions have been investigated by measuring ion transportation in vitro and in vivo under physiological or pathological conditions (7). CLDN2 and 15 are well known cation-selective channel proteins $(8,9)$. Additionally, CLDN10a is anion-selective while $10 \mathrm{~b}$ is cation-selective (6). When CLDN10b is more widely distributed in epithelial cells in various tissues, CLDN10a is only detected in the uterus and kidney (10). A number of the CLDNs (CLDN4, 7, 8 and 16) have inconsistent ion selectivity, and they function as ion-selective channels and sealing proteins (9). CLDN1 and 2 are relatively well studied (2,11-13), whereas, the roles of other CLDNs, including CLDN4, 7, 8 and 16, remain to be defined due to inconsistent results (9). In particular, CLDN4 exhibits varying functions according to the cell type observed. For example, it functions as a sodium barrier when overexpressed in MDCK cells, whereas it acts as a sodium pore during knockdown in M-1 and mIMCD3 cells $(14,15)$. This inconsistency implies that the function of CLDN4 is correlated with interactions with other tight junction proteins.

Previous studies have analyzed the expression and function of CLDNs in various tissues and species. In the present study, the distribution of well-known CLDN family members was examined in order to compare the expression and correlation with other CLDN proteins.

The epithelial and endothelial cells are positive regions and are a novel location in which ion permeability could be more directly controlled by the action of a tight junction. 
Table I. Primer list for claudins (CLDNs) used in the present study.

\begin{tabular}{lll}
\hline Claudin & Forward & Reverse \\
\hline CLDN1 & 5'-AGGTCTGGCGACATTAGTGG-3' & 5'-CGTGGTGTTGGGTAAGAGGT-3' \\
CLDN2 & 5'-TGGTTCCTGACAGCATGAAA-3' & 5'-CTTTGGGCTGTTGAGCAGAT-3' \\
CLDN3 & 5'-GAGATGGGAGCTGGGTTGTA-3' & 5'-ACGTAGTCCTTGCGGTCGTA-3' \\
CLDN4 & 5'-ATCGTTGTCCGCGAGTTCTA-3' & 5'-GCTTGTCGTTGCTACGAGGT-3' \\
CLDN5 & 5'-GCTGGTGGCACTCTTTGTTA-3' & 5'-GCACCGTCGGATCATAGAAC-3' \\
CLDN7 & 5'-TTTCATTGTGGCAGGTCTTG-3' & 5'-TTGCTTTCACTGCCTGGAC-3' \\
CLDN8 & 5'-ATGCAGTGCAAGGTCTACGA-3' & 5'-AGCCGGTGATGAAGAAGATG-3' \\
CLDN10a & 5'-TCCAACGAATGGAAAGTGACC-3' & 5'-TCTCCTTCTCCGCCTTGATAC-3' \\
CLDN10b & 5'TCTCCTTCTCCGCCTTGATAC-3' \\
CLDN11 & 5'-TCGCCTTCGTAGTCTCCATC-3' & 5'-GTGGGCACATACAGGAAACC-3' \\
CLDN12 & 5'-CAGGCTTGTAGAGCCCTCAT-3' & 5'-CGTGATGAATAGGGCTGTGA-3' \\
CLDN14 & 5'-AGGAAGTTTGAGCCGGTCTT-3' & 5'-CATTCAGCCTGTACCCACTGT-3' \\
CLDN15 & 5'-CTGGGCTTCATCTCCTCATC-3' & 5'-TCCAGCATACAGTGGGTTGA-3' \\
CLDN16 & 5'-GCCTCTTTCTAGGCATGGTG-3' \\
CLDN17 & 5'-CTTGGCCATATTCTCCACTG-3' & 5'-GAGTCGTACTCATCGCAGGT-3' \\
CLDN19 & 5'-ATTCCAGTGTCCTGGACTGC-3' & 5'-ATGCAGGGACTGGGTATCTG-3'
\end{tabular}

In order to investigate the expression of CLDNs in various tissues, the quantification of CLDN genes, including CLDN3, $4,5,7,8,10 a, 10 b, 11,14,15$ and 17 , was performed. Notably, the majority of CLDN transcripts were detected in various tissues. Since these observations were noteworthy, the distribution and expression of several CLDN proteins, CLDN1, 2, 12 , and 16 that are responsible for ion transport, in particular calcium, in the intestine and kidney $(16,17)$ were intensively investigated.

\section{Materials and methods}

Animals and tissues. Five age-matched (age, 9-10 weeks) male C57BL/6 mice were obtained from Koatech (Pyeongtaek, Gyeonggi, Korea). All the mice were euthanized by ether, and tissue samples from the duodenum, ileum, colon, kidney, liver and lung were obtained. These samples were used for quantitative polymerase chain reaction (qPCR), western blotting and immunohistochemistry analysis. The Ethics Committee of Chungbuk National University (Cheonju, Korea) approved all the experimental procedures.

$R N A$ extraction and $q P C R$. In order to extract the total RNA, TRIzol reagent (Ambion, Austin, TX, USA) was used according to the manufacturer's instructions. The total RNA concentration was determined by measuring the absorbance at $260 \mathrm{~nm}$ by EPOCH (BioTek Instruments, Inc., Winooski, VT, USA). To synthesize the first-strand complementary DNA (cDNA), $1 \mu \mathrm{g}$ total RNA was reverse-transcribed using moloney murine leukemia virus reverse transcriptase (1:1,000; Invitrogen Life Technologies, Carlsbad, CA, USA) along with random primers (9-mers; Takara Bio, Inc., Otsu, Shiga, Japan). Reverse transcription-PCR was performed on a 7300 Real-Time PCR system (Applied Biosystems, Foster City, CA, USA) according to the manufacturer's instructions. $\beta$-actin was used for normalization and relative gene expression levels were quantified using RQ software (Applied Biosystems). The primer sequences used are listed in Table I.

qPCR reactions contained $1 \mu \mathrm{l}$ cDNA template with $10 \mu \mathrm{l}$ of 2X SYBR Premix Ex Taq (Takara Bio, Inc.) and 10 pmol specific primers. Each reaction was conducted over 40 cycles of denaturation at $95^{\circ} \mathrm{C}$ for $15 \mathrm{sec}$, annealing at $62^{\circ} \mathrm{C}$ for $15 \mathrm{sec}$, and extension at $72^{\circ} \mathrm{C}$ for $30 \mathrm{sec}$. The fluorescence intensity was measured at the end of the extension phase of each cycle , and was performed using an ABI Prism 7300 Sequece 10 detections system (Applied Biosystem) equipped with a 96-well optical reaction plate. Threshold values for the fluorescence intensity of the samples were manually set. A threshold cycle was defined as the cycle when sample fluorescence reached the threshold value. For normalization purposes, $\beta$-actin was used as a control.

Western blotting. Protein extraction was performed using radioimmunoprecipitation assay (RIPA) buffer [50 $\mathrm{mM}$ Tris-HCl (Amresco Inc., Solon, OH, USA), 1\% NP-40 (Calbiochem, Darmstadt, Germany), $150 \mathrm{mM} \mathrm{NaCl}$ (Duchefa Biochemie, Haarlem, The Netherlands), 1 mM EDTA (Boehringer, Mannheim, Germany) and 2 protease inhibitor cocktail tablets (Roche Diagnostics, Mannheim, Germany) in $50 \mathrm{ml}$ of total RIPA solution] and homogenized. Following centrifugation at $13,000 \mathrm{x} \mathrm{g}$, each protein (20 $\mu \mathrm{g} /$ lane) was separated by $10 \%$ SDS-PAGE and transferred to nitrocellulose membranes (Millipore, Bedford, MA, USA). The membranes were then blocked with 5\% skimmed milk (Merck, Darmstadt, Germany), resolved in Tris-buffered saline with Tween-20 (TBS-T) for $2 \mathrm{~h}$ at room temperature, and then incubated overnight with the following primary antibodies at $4^{\circ} \mathrm{C}$ : Mouse anti-CLDN1, CLDN2 and CLDN5 (1:1,000; Invitrogen Life Technologies), rabbit anti-CLDN4, 12 and 16, (1:1,000; Invitrogen Life Technologies), rabbit anti-CLDN19 (1:1,000; Santa Cruz Biotechnology, Inc., Santa Cruz, CA, USA), or rabbit anti- $\beta$-actin (1:1,000; Santa Cruz Biotechnology, Inc.) 
Table II. Relative mRNA expression levels of CLDNs.

\begin{tabular}{|c|c|c|c|c|c|c|}
\hline \multirow[b]{2}{*}{ Claudin } & \multicolumn{6}{|c|}{ Organ-specific claudin expression } \\
\hline & Duodenum & Ileum & Colon & Kidney & Liver & Lung \\
\hline CLDN1 & 100.00 & 110.00 & 240.00 & 8300.00 & 38670.00 & 2140.00 \\
\hline CLDN2 & 100.00 & 100.00 & 90.00 & 1380.00 & 20.00 & 10.00 \\
\hline CLDN3 & 100.00 & 100.00 & 290.00 & 20.00 & 100.00 & 80.00 \\
\hline CLDN4 & 100.00 & 150.00 & 90.00 & 80.00 & 2.00 & 14.00 \\
\hline CLDN5 & 100.00 & 310.00 & 350.00 & 1080.00 & 550.00 & 25060.00 \\
\hline CLDN7 & 100.00 & 130.00 & 160.00 & 8.20 & 0.20 & 10.00 \\
\hline CLDN8 & 100.00 & 370.00 & 12800.00 & 43220.00 & 160.00 & 680.00 \\
\hline CLDN10a & - & - & - & 100.00 & 0.10 & - \\
\hline CLDN10b & 100.00 & 210.00 & 680.00 & 401170.00 & 510.0 & 23750.00 \\
\hline CLDN11 & 100.00 & 300.00 & 210.00 & 11140.00 & 20.00 & 630.00 \\
\hline CLDN12 & 100.00 & 90.00 & 210.00 & 770.00 & 640.00 & 100.00 \\
\hline CLDN14 & 100.00 & 120.00 & 2390.00 & 520.00 & 25730.00 & 460.00 \\
\hline CLDN15 & 100.00 & 42.00 & 43.00 & 5.30 & 0.20 & 2.00 \\
\hline CLDN16 & - & - & - & 100.00 & 0.01 & - \\
\hline CLDN17 & 100.00 & 400.00 & 430.00 & 590.00 & 220.00 & 800.00 \\
\hline CLDN19 & - & - & - & 100.00 & 0.07 & 0.04 \\
\hline
\end{tabular}

Expression levels are marked by the percentage of $2^{-\triangle \Delta C T}$ level. The expression observed in the duodenum was used as a base in each comparison of each CLDN mRNA expression except in CLDN10a, 16 and 19 (where the result of kidney was used as a base). CLDNs, claudins; -, not detected by qPCR. qPCR, quantitative polymerase chain reaction.

overnight at $4^{\circ} \mathrm{C}$. Following the reaction with antibodies, the membranes were washed with TBS-T for $1 \mathrm{~h}$ at room temperature and incubated with anti-rabbit horseradish peroxidase-conjugated secondary antibody (1:3,000; Santa Cruz Biotechnology, Inc.) for $2 \mathrm{~h}$ at room temperature. Subsequent to washing with TBS-T again, antibody binding was detected using an enhanced chemiluminescence reagent (Amersham Biosciences, Little Chalfont, UK), and was recorded by exposure to Biomax ${ }^{\mathrm{TM}}$ Light film (Kodak Express, London, UK) for 1-30 min.

Immunohistochemistry. Tissue-specific localizations of CLDNs were examined by immunohistochemistry. The samples of the duodenum, ileum, colon, kidney, liver and lung were embedded in paraffin, cut into 5- $\mu \mathrm{m}$ sections, mounted onto slides glasses (Matunami, Ishikawa, Japan) coated with amino silane, deparaffinized using xylene and then hydrated in a descending graded ethanol series. The endogenous peroxidase activity was blocked by incubation with $3 \%$ hydrogen peroxidase in phosphate-buffered saline (PBS) for $30 \mathrm{~min}$ at room temperature. Non-specific reaction of sections was blocked by incubation with $10 \%$ goat serum (Vector Laboratories, Inc. Burlingame, CA, USA) in PBS for $1 \mathrm{~h}$ at room temperature. Subsequent to washing using TBS-T, the sections were reacted with biotinylated secondary antibodies (1:500, rabbit or mouse IgG; Vector Laboratories, Inc.) for $1 \mathrm{~h}$ at $37^{\circ} \mathrm{C}$, followed by incubation with ABC-Elite (Vector Laboratories, Inc.) for $30 \mathrm{~min}$ at $37^{\circ} \mathrm{C}$. Diaminobezidine (Sigma, St. Louis, MO, USA) was used as a chromogen. The sections were then counterstained with

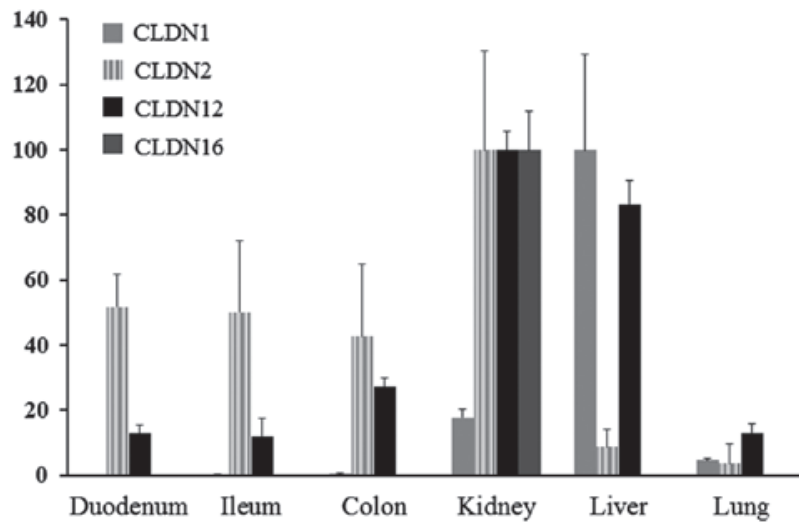

Figure 1. Tissue-specific mRNA expression of CLDNs in mouse tissues. The mRNA expression of CLDN1, 2, 12 and 16 in the duodenum, ileum, colon, kidney, liver and lung was investigated by qPCR. CLDN mRNA expression in each tissue was shown as the percentage of relative expression compared with the that of the tissue with the highest expression of each CLDNs (CLDN1: liver, CLDN2, 12 and 16: kidney). CLDNs, claudins; qPCR, quantitative polymerase chain reaction.

hematoxylin and mounted in CytoSeal ${ }^{\mathrm{TM}} 60$ (Richard-Allan Scientific Co., Kalamazoo, MI, USA).

\section{Results}

Tissue-specific mRNA expression levels of CLDNs. The tissue-specific mRNA expression levels of CLDNs (1-5, 7-8, 10 $a$ and $b, 11-12,14-17$ and 19) in the duodenum, ileum, colon, kidney, liver and lung were analyzed by qPCR 


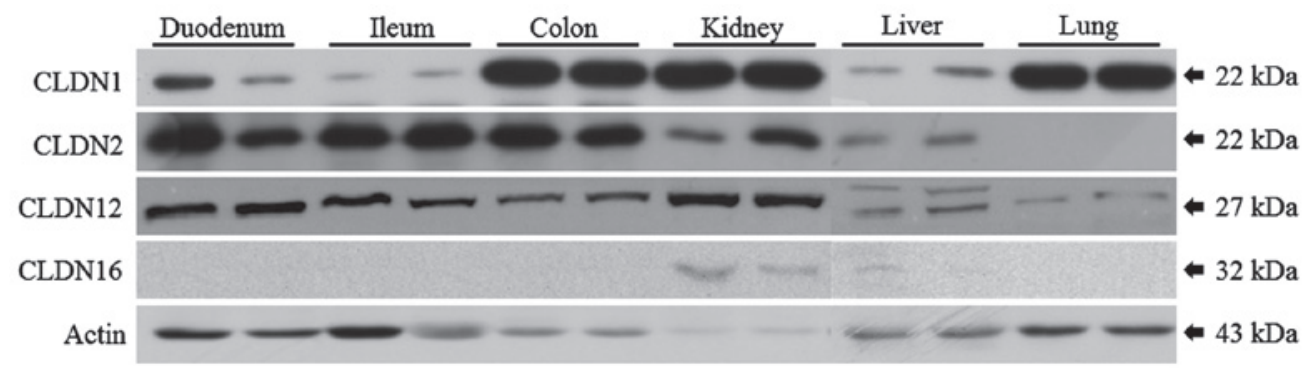

Figure 2. Western blotting was used to analyze claudin (CLDN) protein expression in the duodenum, ileum, colon, kidney, liver and lung. The results were normalized relative to that of actin.

(Table II). CLDN1 expression was highest in the liver and moderately higher in the kidney and lung compared to other tissues (Fig. 1). CLDN2, 3, 4, 5 and 12 were highly expressed overall. Specifically, CLDN2 expression was highest in the kidney, similar in the duodenum, ileum and kidney and relatively lower in the liver and lung (Fig. 1). CLDN3 expression was highest in the colon, similar in the duodenum, ileum and liver, and relatively low in the kidney. CLDN4 expression was highest in the ileum, and relatively lower in the liver and lung. CLDN5 was extremely highly expressed in the lung compared with the other tissues. CLDN12 was relatively highly expressed in the kidney and liver (Fig. 1). The mRNA expression of CLDN7 was higher in the small and large intestines compared with that in the other organs, although it was highest in the colon. CLDN8 mRNA expression was highly expressed in the colon and kidney compared with the tissues. The mRNA levels of CLDN11 were the highest in the kidney, and CLDN14 transcripts were the highest in the liver. The relative expression of CLDN10a and 16 mRNA in various tissues was monitored, and they were shown to be highly expressed in the kidney compared with that of any other tissues, less expressed in the liver than that of the kidney, and not expressed in the others. The CLDN1Ob mRNA distribution pattern was different from that of CLDN10a; it was expressed in all the tested tissues and highly expressed in the kidney compared with the others. The CLDN17 mRNA expression in the lung was higher compared with the other tissues. The CLDN19 mRNA expression was highest in the kidney and relatively high in the liver compared with the lung and the expression of CLDN19 mRNA was not detected in the intestine.

Tissue-specific translational expression of CLDNs. The protein levels of CLDN1, 2, 12 and 16 were analyzed in the duodenum, ileum, colon, kidney, liver and lung by western blotting (Fig. 2). The CLDN1 protein expression was highest in the kidney and relatively higher in the lung compared with the duodenum, ileum and liver. The CLDN2 protein distribution was relatively high in the duodenum, ileum and colon compared with the kidney, whereas it was absent from the lung. The protein expression of CLDN12 was detected in all tested tissues; it was higher in the kidney and colon compared with the liver and lung. The protein distribution of CLDN16 was similar to its mRNA distribution; it was only expressed in the kidney and liver and was highest in the kidney. The expression of CLDN4 and 19 proteins was not detectable.
Tissue-specific localization of CLDNs. CLDNs were commonly expressed in erythrocytes of the small/large intestine, hepatocytes of the liver and epithelial cells of teh bronchium. Tissue-specific localization of CLDN1, 2, 12 and 16 in the duodenum, ileum, colon, kidney, liver and lung were investigated by immunohistochemistry (Fig. 3). Localization of CLDN1 and 12 were detected in all the tested tissues (Fig. $3 \mathrm{~A}$ and $\mathrm{C}$ ). CLDN2 was localized to the duodenum, ileum, colon, kidney and liver (Fig. 3B). However, the localization of CLDN16 was observed in the kidney and liver only (Fig. 3D). In the kidney, each CLDN localization pattern was different. CLDN1 was localized to Bowman's capsules, proximal tubules and the distal nephron. CLDN2 and CLDN12 were detected in the proximal tubules. CLDN16 was positioned in the thin and thick ascending limbs of the loop of Henle. Immunohistochemistry was also performed for CLDN4 and CLDN19, but immune-positive staining was not detected.

\section{Discussion}

Tight junctions consist of transmembrane proteins, of which CLDNs are a major type. CLDNs are representative transmembrane proteins, and are responsible for barrier and selective ion transport functions of tight junctions. In the present study, the expression and distribution of CLDNs in various tissues, including the duodenum, ileum, colon, kidney, liver and lung, was examined. The mRNA expression of 16 CLDNs (1-5, 7-8, 10a and b, 11-12, 14-17 and 19) was tested. Among the tested CLDNs, CLDN1, CLDN2, CLDN12 and CLDN16 were notably expressed in a tissue-dependent manner, and therefore the protein expression and localization of those CLDNs was analyzed further.

The mRNA expression of CLDN1 was highest in the liver and relatively high in the kidney, lung and muscle. However, CLDN1 protein was highest in the kidney and moderate in the colon and lung. Unlike its mRNA distribution, the CLDN1 protein expression was low in the liver. The localization of CLDN1 was confirmed as previously reported. CLDN1 is known to function in sealing junctions without ion selectivity (12). The presence of sealing CLDNs render tight junctions more 'tight', thus they are highly expressed in tight rather than leaky epithelial cells, which are distinguished by their electrical resistance (9). CLDN1 was investigated as it was shown to be correlated with skin defects in a knockout study in mice, and was also shown to be capable of enhancing the ability of the tight junction in MDCK cells in an 

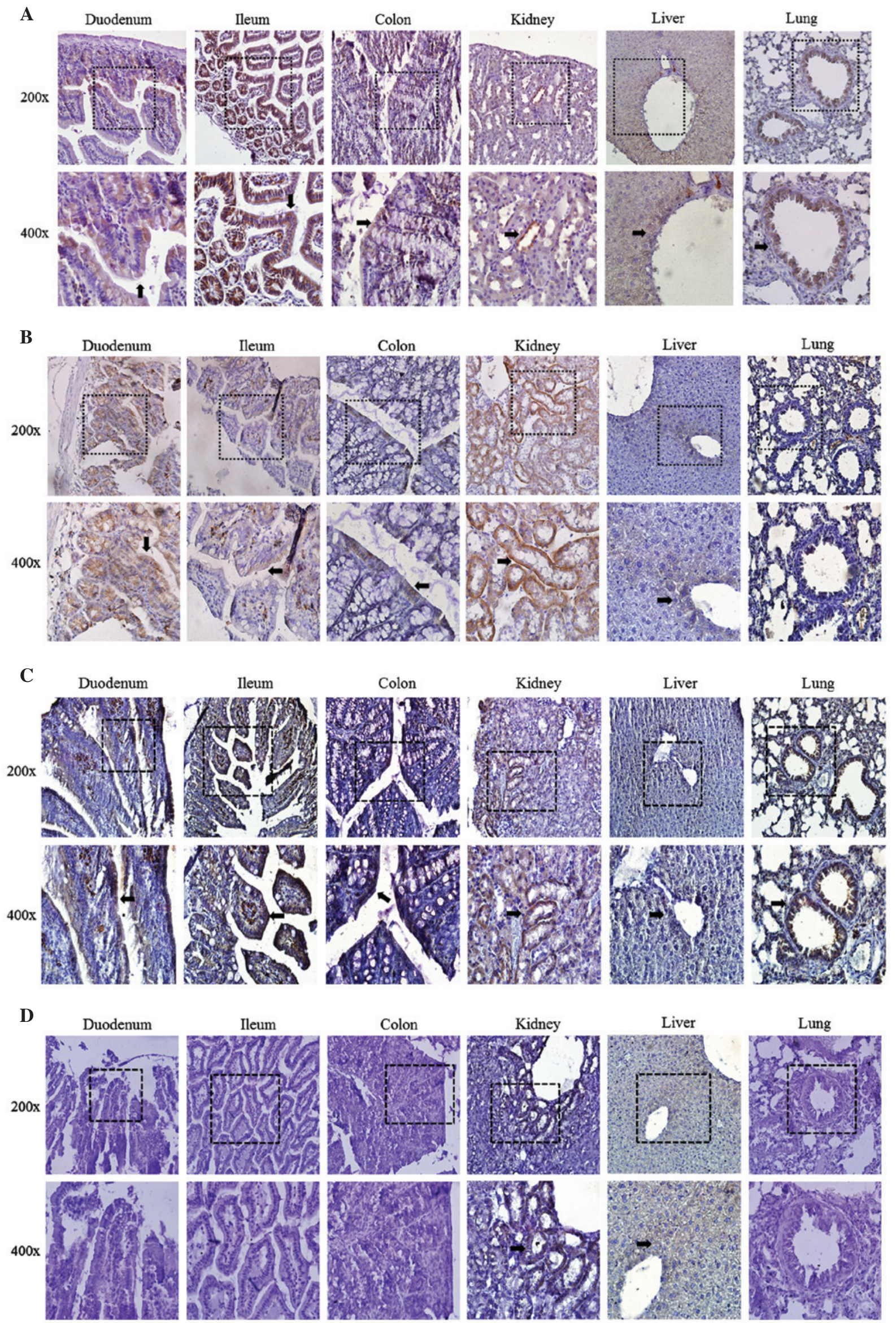

Figure 3. Tissue-specific localization of claudins (CLDNs) in mice tissues. (A) Localizations of CLDN1, (B) CLDN2, (C) CLDN12 and (D) CLDN16 in the duodenum, ileum, colon, kidney, liver and lung were assessed by immunohistochemistry. The black arrow indicates each region positively stained for CLDN. Mouse anti- CLDN1, 2 and Rabbit anti- CLDN12, 16 were used for staining, and hematoxylin was used for counterstaining. Each figure was magnified as $\mathrm{x} 200$ and $\mathrm{x} 400$. 
overexpression study (12). CLDN1 has also been shown to act as a tumor suppressor gene in breast tumor $(11,18)$.

CLDN2 is known as a cation-selective channel-forming protein (2). Expression of CLDN2 has been reported in various tissues, including the intestine, kidney, human ovarian surface epithelium and inner ear $(19,20)$. In the present study, CLDN2 mRNA was generally distributed in all the tested tissues. Specifically, expression of CLDN2 was highest in the kidney, relatively high in the duodenum, ileum, colon and liver, and relatively low in the lung. However, CLDN2 protein was not detected in the lung, whereas its expression was low in the liver and relatively high in the kidney, duodenum, ileum and colon. CLDN2 is responsible for calcium absorption in the enterocytes of the intestine, and it increases paracellular cation permeability in the kidney $(2,16,21)$. In contrast to CLDN1, CLDN2 expression has been reported in leaky epithelial cells, including those of the renal proximal tubules, intestinal crypts and choroid plexus, rather than in tight epithelial cells (22-24). It is therefore of note that CLDN2 has cation selectivity by the charged amino acids in the first extracellular loop in its structure. It has been revealed that CLDN2 and 12 are critically correlated with vitamin D-dependent calcium absorption between enterocytes (12), and that CLDN2 knockout mice are defective in the leaky- and cation-selective paracellular permeability properties of the renal proximal tubules $(16,25)$. Although the CLDN2 transcript was highly expressed in the liver, its protein levels were lower compared with that in other somatic tissues.

Among all the tested CLDN mRNAs, CLDN12 mRNA was most equally distributed, although it was highest in the kidney and moderately high in the liver. The levels of CLDN12 mRNA were well expressed in all the tested tissues, whereas its proteins were differentially expressed. CLDN12 expression was found in the blood-brain barrier with CLDN3 and 5 (26). Another study has reported that CLDN12 is suppressed by hyper-ammonemia in brain capillary endothelial cells (27), and it was detected in various tissues, including the intestine, urinary bladder and epidermis $(28,29)$. The expression of CLDN12 has predominantly been reported in tight junctions, in which it functions as a barrier between cells (9). Nevertheless, CLDN12 is responsible for calcium absorption in the intestine since it forms a calcium-selective channel in intestinal enterocytes (16).

CLDN16 mRNA expression was expressed only in the kidney and liver, and it was highest in the kidney. The level of CLDN16 protein was parallel with that of its transcript, and it was localized in the renal distal convoluted tubule. Previous reports have revealed that renal hypomagnesemia was linked with a mutation in CLDN16 in the kidney (30,31). CLDN16 has been reported to form cation channels; however, the latter could not be confirmed in another study (9). Moreover, attempts to explain the pathophysiology of magnesium transport and to define the role of CLDN16 have been unsuccessful (17). Although previous studies are insufficient to define the functions of CLDN16, it is certain that the interactions of CLDN16 with other proteins are critical for calcium and magnesium homeostasis in the body (17). In conclusion, the present study on mouse tissue indicated that CLDNs were expressed in the intestine, kidney, liver and lung. An immunohistochemical study also revealed that the majority of the CLDN subtypes were located in the epithelium of certain tissues. These results indicated that CLDNs are expressed and localized in a tissue-specific manner, which may suggest functional roles of CLDNs in the tissues. Furthermore, the results of this study may aid in the investigation of physiological disorders linked with mutations or the overexpression of CLDNs.

\section{Acknowledgements}

This study was supported by the National Research Foundation of Korea (NRF) grant funded by the Korea government (MEST) (grant no. 2013R1A2A2A05004582).

\section{References}

1. Wen H, Watry DD, Marcondes MC and Fox HS: Selective decrease in paracellular conductance of tight junctions: role of the first extracellular domain of claudin-5. Mol Cell Biol 24: 8408-8417, 2004.

2. Amasheh S, Meiri N, Gitter AH, et al: Claudin-2 expression induces cation-selective channels in tight junctions of epithelial cells. J Cell Sci 115: 4969-4976, 2002.

3. Hou J, Paul DL and Goodenough DA: Paracellin-1 and the modulation of ion selectivity of tight junctions. J Cell Sci 118: 5109-5118, 2005.

4. Colegio OR, Van Itallie C, Rahner C and Anderson JM: Claudin extracellular domains determine paracellular charge selectivity and resistance but not tight junction fibril architecture. Am J Physiol Cell Physiol 284: C1346-C1354, 2003.

5. Alexandre MD, Jeansonne BG, Renegar RH, Tatum R and Chen YH: The first extracellular domain of claudin-7 affects paracellular $\mathrm{Cl}$ permeability. Biochem Biophys Res Commun 357: 87-91, 2007.

6. Van Itallie CM, Rogan S, Yu A, Vidal LS, Holmes J and Anderson JM: Two splice variants of claudin-10 in the kidney create paracellular pores with different ion selectivities. Am J Physiol Renal Physiol 291: F1288-F1299, 2006.

7. Van Itallie CM and Anderson JM: Claudins and epithelial paracellular transport. Annu Rev Physiol 68: 403-429, 2006.

8. Krug SM, Günzel D, Conrad MP, et al: Charge-selective claudin channels. Ann NY Acad Sci 1257: 20-28, 2012.

9. Schulzke JD, Günzel D, John LJ and Fromm M: Perspectives on tight junction research. Ann NY Acad Sci 1257: 1-19, 2012.

10. Günzel D, Stuiver M, Kausalya PJ, et al: Claudin-10 exists in six alternatively spliced isoforms that exhibit distinct localization and function. J Cell Sci 122: 1507-1517, 2009.

11. Myal Y, Leygue E and Blanchard AA: Claudin 1 in breast tumorigenesis: revelation of a possible novel 'claudin high' subset of breast cancers. J Biomed Biotechnol 2010: 956897, 2010

12. Inai T, Kobayashi J and Shibata Y: Claudin-1 contributes to the epithelial barrier function in MDCK cells. Eur J Cell Biol 78: 849-855, 1999.

13. Weber CR, Nalle SC, Tretiakova M, Rubin DT and Turner JR: Claudin-1 and claudin-2 expression is elevated in inflammatory bowel disease and may contribute to early neoplastic transformation. Lab Invest 88: 1110-1120, 2008.

14. Van Itallie C, Rahner C and Anderson JM: Regulated expression of claudin-4 decreases paracellular conductance through a selective decrease in sodium permeability. J Clin Invest 107: 1319-1327, 2001.

15. Hou J, Renigunta A, Yang J and Waldegger S: Claudin-4 forms paracellular chloride channel in the kidney and requires claudin-8 for tight junction localization. Proc Natl Acad Sci USA 107: 18010-18015, 2010.

16. Fujita H, Sugimoto K, Inatomi S, et al: Tight junction proteins claudin-2 and -12 are critical for vitamin D-dependent $\mathrm{Ca}^{2+}$ absorption between enterocytes. Mol Biol Cell 19: 1912-1921, 2008.

17. Hou J, Renigunta A, Konrad M, et al: Claudin-16 and claudin-19 interact and form a cation-selective tight junction complex. J Clin Invest 118: 619-628, 2008.

18. Chao YC, Pan SH, Yang SC, et al: Claudin-1 is a metastasis suppressor and correlates with clinical outcome in lung adenocarcinoma. Am J Respir Crit Care Med 179: 123-133, 2009.

19. Kitajiri SI, Furuse M, Morita K, et al: Expression patterns of claudins, tight junction adhesion molecules, in the inner ear. Hear Res 187: 25-34, 2004. 
20. Zhu Y, Brännström M, Janson PO and Sundfeldt K: Differences in expression patterns of the tight junction proteins, claudin 1 , 3, 4 and 5, in human ovarian surface epithelium as compared to epithelia in inclusion cysts and epithelial ovarian tumours. Int J Cancer 118: 1884-1891, 2006.

21. Furuse M, Furuse K, Sasaki H and Tsukita S: Conversion of zonulae occludentes from tight to leaky strand type by introducing claudin-2 into Madin-Darby canine kidney I cells. J Cell Biol 153: 263-272, 2001.

22. Reyes JL, Lamas M, Martin D, et al: The renal segmental distribution of claudins changes with development. Kidney Int 62: 476-487, 2002.

23. Rahner C, Mitic LL and Anderson JM: Heterogeneity in expression and subcellular localization of claudins $2,3,4$, and 5 in the rat liver, pancreas, and gut. Gastroenterology 120: 411-422, 2001.

24. Wolburg H, Wolburg-Buchholz K, Liebner S and Engelhardt B Claudin-1, claudin-2 and claudin-11 are present in tight junctions of choroid plexus epithelium of the mouse. Neurosci Lett 307 : $77-80,2001$.

25. Muto S, Hata M, Taniguchi J, et al: Claudin-2-deficient mice are defective in the leaky and cation-selective paracellular permeability properties of renal proximal tubules. Proc Natl Acad Sci USA 107: 8011-8016, 2010
26. Hawkins BT and Davis TP: The blood-brain barrier/neurovascular unit in health and disease. Pharmacol Rev 57: 173-185, 2005.

27. Belanger M, Asashima T, Ohtsuki S, Yamaguchi H, Ito S and Terasaki T: Hyperammonemia induces transport of taurine and creatine and suppresses claudin-12 gene expression in brain capillary endothelial cells in vitro. Neurochem Int 50: 95-101, 2007.

28. Fujita H, Chiba H, Yokozaki H, et al: Differential expression and subcellular localization of claudin-7, $-8,-12,-13$ and -15 along the mouse intestine. J Histochem Cytochem 54: 933-944, 2006.

29. Brandner JM, McIntyre M, Kief S, Wladykowski E and Moll I: Expression and localization of tight junction-associated proteins in human hair follicles. Arch Dermatol Res 295: 211-221, 2003.

30. Simon DB, Lu Y, Choate KA, et al: Paracellin-1, a renal tight junction protein required for paracellular $\mathrm{Mg}^{2+}$ resorption. Science 285: 103-106, 1999

31. Konrad M, Schaller A, Seelow D, et al: Mutations in the tight-junction gene claudin 19 (CLDN19) are associated with renal magnesium wasting, renal failure, and severe ocular involvement. Am J Hum Genet 79: 949-957, 2006. 